\title{
RELAÇÃO ENTRE NÍVEL DE ATIVIDADE FÍSICA, CONDIÇÕES DE SAÚDE E OCUPACIONAIS ENTRE BOMBEIROS MILITARES
}

\author{
Bianca Pereira de JESUS ${ }^{1}$ \\ Luis Henrique Ferreira dos REIS ${ }^{2}$ \\ Idael Emiliano Gomes SILVA ${ }^{3}$ \\ Danilo Lima CARREIRO ${ }^{4}$ \\ Laura Tatiany Mineiro COUTINHO \\ Luciana Caldeira de Paula RICARDO ${ }^{6}$ \\ Andréa Maria Eleutério de Barros Lima MARTINS ${ }^{7}$ \\ Wagner Luiz Mineiro COUTINHO ${ }^{8}$
}

Recebido em: 09/11/2014 - Aprovado em: 03/06/2015 - Disponibilizado em: 15/07/2015

${ }^{1}$ Estudante do Curso de Graduação em Fisioterapia da Associação Educativa do Brasil (SOEBRAS),

bibi-pj@hotmail.com

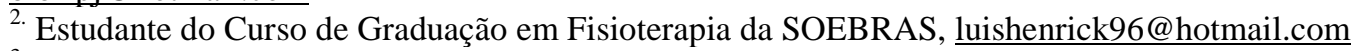

3. Pós-graduado em Fisioterapia Ortopédica e Postural (SOEBRAS). Docente da SOEBRAS, idaelemiliano@yahoo.com.br

4. Mestre em Ciências da Saúde pela Universidade Estadual de Montes Claros (Unimontes). Docente do Instituto Federal de Educação, Ciência e Tecnologia do Norte de Minas (IFNMG). Campus Pirapora, danilolimacarreiro@gmail.com

${ }^{5 .}$ Mestre em Ciências da Saúde pela Unimontes. Docente da SOEBRAS, mineiro.laura@ gmail.com

6. Mestre em Ciência e Tecnologia de Alimentos pela Universidade Federal de Viçosa (UFV). Docente da SOEBRAS, lucianaricardo@gmail.com

7. Doutora em Saúde Pública (Epidemiologia) pela Universidade Federal de Minas Gerais (UFMG). Docente da Unimontes e da SOEBRAS, martins.andreamebl@gmail.com

${ }^{8}$ Mestre em Ciências da Saúde pela Unimontes. Professor da SOEBRAS, coutinhowlm@gmail.com

RESUMO: este estudo teve por objetivo determinar a prevalência do nível de atividade física entre bombeiros militares e sua relação com condições demográficas, socioeconômicas, de saúde e ocupacionais. Trata-se de um estudo transversal analítico no qual se utilizaram para coleta de dados os questionários: International Physical Activity Questionnaire, Maslach Burnout Inventory, WHOQOL-Bref, Critério de Classificação Econômica Brasil e Questionário demográfico-socioeconômico, condições de saúde e laborais. Identificou-se que 7,4\% $(\mathrm{n}=15)$ dos bombeiros foram classificados como sedentários; $15,3 \%(\mathrm{n}=31)$ como irregularmente ativo A; 7,0\% ( $\mathrm{n}=14)$ como irregularmente ativo B; 20,3\% ( $n=41)$ como ativo e 50,0\% $(n=101)$ como muito ativo. Através de regressão logística múltipla registrou-se maior chance de baixo nível de atividade física entre bombeiros com baixa percepção do nível de qualidade de vida no domínio físico quando comparados aos bombeiros com alta percepção do nível de qualidade de vida neste domínio (OR: 2,$06 ; \mathrm{IC}_{95 \%}: 1,10-3,86 ; p=0,023$ ) e entre bombeiros que relataram ter posse de outro emprego quando comparados àqueles que afirmaram exercer apenas a atividade laboral militar (OR: 2,14; ICIC $_{95 \%}$ : 1,02-5,32; $\left.p=0,049\right)$. A prevalência de inatividade física esteve presente em aproximadamente um terço dos bombeiros sendo que, o baixo nível de atividade física relacionou-se à baixa percepção do nível de qualidade de vida no domínio físico e ao fato do bombeiro ter posse de outro emprego.

Palavras-chave: Atividade motora. Condições de saúde. Condições de trabalho. Bombeiros. Saúde do trabalhador.

\section{RELATIONSHIP BETWEEN LEVEL OF PHYSICAL ACTIVITY, AND OCCUPATIONAL HEALTH AMONG FIREFIGHTERS}

\begin{abstract}
: the objective of this study was to determine the prevalence of physical activity among firefighters and their relationship with demographic, socioeconomic, health and occupational conditions. This is an analytical crosssectional study and it was used for data collection questionnaires: International Physical Activity Questionnaire, Maslach Burnout Inventory, WHOQOL-Bref, Critério de Classificação Econômica Brasil, and demographicsocioeconomic, health and labor questionnaire. It was found that $7.4 \%(n=15)$ firemen were classified as sedentary; $15.3 \%(n=31)$ as irregularly active $A ; 7.0 \%(n=14)$ as irregularly active $B ; 20.3 \%(n=41)$ as active and $50.0 \%(n=101)$ as very active. By multiple logistic regression was registered higher chance of low level of physical activity among
\end{abstract}


firefighters with low perceived level of quality of life in the physical domain compared to firefighters with high perceived level of quality of life in this area (OR: 2.06; CI95\%: 1.10 to 3.86; $\mathrm{p}=0.023$ ) and among firefighters who reported having possession of another job compared to those who reported exercising only such military occupational activity (OR: 2.14; CI 95\%: 1.02 to 5.32; $\mathrm{p}=0.049$ ). The prevalence of physical inactivity was present in approximately one third of firefighters and that the low level of physical activity was related to lower perceived level of quality of life in the physical domain and the fireman have possession of another job.

Keywords: Motor activity. Health status. Working conditions. Firefighters. Occupational health.

\section{INTRODUÇÃO}

A atividade física (AF) é considerada pela Organização Mundial de Saúde (OMS) como um dos fatores de proteção em relação às doenças e agravos não transmissíveis (DANTs) possivelmente evitáveis (PAHO, 2007). Dentre as ações benéficas de sua prática regular se encontram: controle do estado nutricional (DA MOTA E ZANESCO, 2007), dos níveis pressóricos (ZAGO e ZANESCO, 2006) e do estresse e fadiga laboral (BARRETO e BARBOSA-BRANCO, 2000); prevenção de doenças cardiovasculares e metabólicas (ACSM, 2009); e impacto positivo na percepção do nível de qualidade de vida (QV) (MACEDO et al., 2003).

Todavia, o estilo de vida contemporâneo caracterizado por mecanização, avanços tecnológicos, informatização, transportes motorizados, mecanismos que poupam esforço físico (labor saving devices) como escadas rolantes, elevadores e dispositivos remotos e ainda pelo lazer hipocinético (assistir televisão, uso de computadores e vídeo games) compromete cada vez mais a prática regular de AF, seja no âmbito de lazer, doméstico ou laboral (NAHAS, BARROS e FRANCALACCI,
2000; JESUS e JESUS, 2012). A este contexto somam-se ainda condições laborais deletérias demarcadas por indisponibilidade temporal para lazer e atividades familiares; degradação do poder econômico; inacessibilidade a bens e serviços essenciais; exposição a agentes estressores e ao sedentarismo (SALLIS e OWEN, 1999).

Ao se considerar os policiais militares, inclusive os bombeiros, um nível favorável de prática de $\mathrm{AF}$ é necessário para o cumprimento laboral uma vez que o sedentarismo pode implicar nestes profissionais adoecimento e comprometimento da vitalidade e da eficiência profissional (MINAYO, ASSIS e OLIVEIRA, 2011; JESUS e JESUS, 2012). Todavia, estudo entre policiais civis e militares do Rio de Janeiro - RJ identificou média do nível de atividade física (NAF) inferior à encontrada entre outras categorias profissionais (MINAYO, ASSIS e OLIVEIRA, 2011). Pesquisa qualitativa entre bombeiros de São Leopoldo - RS constatou que a inatividade física nesta categoria profissional pode sofrer influência social, uma vez que para a sociedade o bombeiro não deve praticar atividades físicas no quartel durante 
atividade laboral, mas sim estar em estado de prontidão enquanto aguarda chamados de socorro (MONTEIRO et al., 2007).

A prática de AF bem como dos fatores relacionados a esta entre bombeiros militares, tem sido pouco estudada na América Latina. Constata-se tal fato ao buscar nas bases de dados Biblioteca Virtual em Saúde (Bireme), Scientific Electronic Library Online (Scielo) e Latin American and Caribbean Health Sciences (LILACS) publicações utilizando os descritores "bombeiros" e "atividade física" e identificar o registro de apenas uma pesquisa, de abordagem qualitativa. Desta forma, com o intuito de contribuir para melhor compreensão de tal prática nesta categoria profissional, este estudo teve por objetivo determinar a prevalência do nível de atividade física e sua relação com condições demográficas, socioeconômicas, de saúde e ocupacionais entre bombeiros militares.

\section{METODOLOGIA}

Estudo transversal censitário entre militares de um batalhão do corpo de bombeiros de uma cidade de grande porte no estado de Minas Gerais. A coleta dos dados ocorreu entre maio e junho de 2012, por meio de entrevistas estruturadas, avaliação antropométrica e dos níveis pressóricos, por pesquisadores examinadores previamente treinados e calibrados. O período matutino, preferencialmente antes do início das atividades ocupacionais, foi priorizado para a coleta de dados, com o intuito de melhor controle da qualidade da antropometria e dos níveis pressóricos.

Considerou-se como variável dependente, o NAF avaliado pelo International Physical Activity Questionnaire (IPAQ). Sua versão curta foi validada para uso no Brasil por Pardini et al., (2001) sendo sua versão original proposta pelo Grupo Internacional para Consenso em Medidas da Atividade Física, constituído sob a chancela da OMS para estimar o nível de prática habitual de atividade física de populações de diversos países e contextos socioculturais. Tal questionário permite estimar o tempo despendido por semana em diferentes dimensões de atividade física que compreendem caminhadas e esforços físicos de intensidades moderada e vigorosa (PARDINI et al., 2001) podendo a pessoa ser classificada em: muito ativa, ativa, irregularmente ativa $\mathrm{A}$, irregularmente ativa $\mathrm{B}$ e sedentária (DA SILVA, FARAH e RICARTE, 2012). As variáveis independentes foram classificadas em variáveis individuais (demográficas, socioeconômicas e de saúde) e variáveis ocupacionais.

Na Tabela 1 são apresentadas as categorizações das variáveis em estudo. 
Tabela 1 - Categorizações das variáveis, relação entre nível de atividade física, condições de saúde e ocupacionais entre bombeiros militares, interior de MG, $2012(n=202)$

\begin{tabular}{|c|c|c|}
\hline Variáveis & Categorização & $\begin{array}{c}\text { Referência } \\
\text { categorização }\end{array}$ \\
\hline \multicolumn{3}{|l|}{ Dependente } \\
\hline Nível de atividade física - NAF & $0=$ fisicamente ativo $1=$ fisicamente inativo ${ }^{a}$ & a \\
\hline \multicolumn{3}{|l|}{ Independentes } \\
\hline \multicolumn{3}{|l|}{ Individuais } \\
\hline \multicolumn{3}{|l|}{ Demográficas } \\
\hline Sexo & $0=$ masculino $1=$ feminino & $\mathrm{b}$ \\
\hline Idade & $0=\leq 27$ anos e $1=>27$ anos (menor quartil) & b \\
\hline Raça ou cor da pele ${ }^{c}$ & $0=$ branca/amarela $1=$ preta/parda/indígena & - \\
\hline \multicolumn{3}{|l|}{ Socioeconômicas } \\
\hline Estado civil ${ }^{\mathrm{c}}$ & $0=$ solteiro $1=$ não solteiro ${ }^{\mathrm{d}}$ & e \\
\hline Segmentação econômica ${ }^{\mathrm{f}}$ & $0=$ baixa $(\mathrm{C} 1 / \mathrm{E}) 1=$ alta $(\mathrm{A} 1 / \mathrm{B} 2)^{\mathrm{g}}$ & h \\
\hline Escolaridade & $0=$ ensino superior $1=$ ensino médio & e \\
\hline \multicolumn{3}{|l|}{ Condições de saúde } \\
\hline Síndrome de Burnout ${ }^{\mathrm{i}}$ & $0=$ ausência $1=$ presença & $\mathrm{j}$ \\
\hline Exaustão emocional $^{\mathrm{i}}$ & $0=$ baixa $/$ moderada $1=$ alta & - \\
\hline Despersonalização $^{\mathrm{i}}$ & $0=$ baixa $/$ moderada $1=$ alta & - \\
\hline Realização profissional $^{\mathrm{i}}$ & $0=$ baixa $/$ moderada $1=$ alta & - \\
\hline Percepção nível QV geral $^{\mathrm{k}}$ & $0=$ alta $1=$ baixa (limite inferior intervalo de confiança) & - \\
\hline Percepção nível QV domínio físico ${ }^{\mathrm{k}}$ & $0=$ alta $1=$ baixa (limite inferior intervalo de confiança) & - \\
\hline Percepção nível QV domínio psicológico ${ }^{\mathrm{k}}$ & $0=$ alta $1=$ baixa (limite inferior intervalo de confiança) & - \\
\hline Percepção nível QV domínio social ${ }^{\mathrm{k}}$ & $0=$ alta $1=$ baixa (limite inferior intervalo de confiança) & - \\
\hline Percepção nível QV domínio ambiental ${ }^{\mathrm{k}}$ & $0=$ alta $1=$ baixa (limite inferior intervalo de confiança) & - \\
\hline Tabagismo & $0=$ não $1=\operatorname{sim}$ & - \\
\hline Consumo de bebidas alcoólicas & $0=$ não $1=\operatorname{sim}$ & - \\
\hline Estado nutricional (IMC) $)^{1^{*}}$ & $0=$ normal $1=$ sobrepeso/obeso & - \\
\hline Índice de Conicidade $(\mathrm{IC})^{\mathrm{m}}$ & $0=$ sem risco $1=$ com risco & - \\
\hline Circunferência Abdominal $(\mathrm{CA})^{\mathrm{n}}$ & $0=$ sem risco $1=$ risco aumentado/risco muito elevado & - \\
\hline Relação Cintura Quadril (RCQ) ${ }^{\circ}$ & $0=$ sem risco $1=$ com risco & - \\
\hline Razão Cintura Estatura (RCEst) ${ }^{\mathrm{p}}$ & $0=$ sem risco $1=$ com risco & - \\
\hline Pressão arterial sistólica e diastólica ${ }^{\mathrm{q}}$ & $0=$ não e $1=\operatorname{sim}$ & - \\
\hline \multicolumn{3}{|l|}{ Ocupacionais } \\
\hline Posse de outro emprego & $0=$ não $1=\operatorname{sim}$ & - \\
\hline Estrutura organizacional & $0=$ administrativo e $1=$ operacional & - \\
\hline Tempo de serviço & $\begin{array}{l}0=>14 \text { anos; } 1=>\text { sete } \mathrm{e} \leq 14 ; 2=>\text { três } \mathrm{e} \leq \text { sete anos; } 3=\leq \\
\text { três anos (quartis) }\end{array}$ & - \\
\hline
\end{tabular}

"Fisicamente Ativo compreendeu "muito ativo" e/ou "ativo" e Fisicamente Inativo compreendeu "irregularmente ativo A e B" e/ou "sedentário" (DA SILVA, FARAH e RICARTE, 2012).

${ }^{\mathrm{b}}$ Malina (2001).

"Investigada de acordo com o "Questionário da Amostra CD 2010”, proposto pelo Instituto Brasileiro de Geografia e Estatística (BRASIL, 2010).

d،solteiro" compreendeu sujeitos solteiros e "não solteiros" compreendeu sujeitos casados e com união estável/divorciados/desquitados ou separados judicialmente/viúvos.

${ }^{\text {e}}$ Pitanga e Lessa (2005).

${ }^{\mathrm{f}}$ Investigada de acordo com o Critério de Classificação Econômica Brasil (CCEB). Tais segmentações representam as respectivas classes de renda familiar: A1: R\$ 12.926,00; A2: R\$ 8.418,00; B1: R\$ 4.418,00; B2: R\$ 2.565,00; C1: R\$ 1.541,00; C2: R\$ 1.024,00; D: R\$ 714,00 e E: R\$ 477,00 (ABEP, 2012).

${ }^{\mathrm{g}}$ Rechenchosky et al. (2009).

${ }^{\mathrm{h}}$ Tribessan, et al. (2009).

i Investigada de acordo com o Maslach Burnout Inventory (MBI), validado para uso no Brasil por Benevides-Pereira (2001).

${ }^{\mathrm{j} S a n t o s}$, Nascimento Sobrinho (2011).

${ }^{\mathrm{k}}$ Investigada de acordo com o Whoqol-Bref, validado para uso no Brasil por Fleck et al. (2000).

${ }^{\mathrm{l}}$ Calculado pela razão peso/altura ${ }^{2}\left(\mathrm{~kg} / \mathrm{m}^{2}\right)$, e posteriormente classificado de acordo com a OMS em índice normal $\left(<25 \mathrm{~kg} / \mathrm{m}^{2}\right)$; sobrepeso (25 a $\left.29,9 \mathrm{~kg} / \mathrm{m}^{2}\right)$ ou obeso $\left(\geq 30 \mathrm{~kg} / \mathrm{m}^{2}\right.$ ) (NASCENTE et al., 2009).

${ }^{\mathrm{m} C o n s i d e r o u ~ c o m o ~ d i s c r i m i n a d o r ~ d e ~ r i s c o ~ e l e v a d o ~ o s ~ p o n t o s ~ d e ~ c o r t e: ~ 1,25 ~ p a r a ~ h o m e n s ~ e ~ 1,18 ~ p a r a ~ m u l h e r e s ~(P I T A N G A ~ e ~ L E S S A, ~}$ 2011).

${ }^{\mathrm{n}}$ Considerou ponto de corte de risco coronariano aumentado para mulheres $\mathrm{CA}>80 \mathrm{~cm}$ e para homens CA $>94 \mathrm{~cm}$, e risco muito aumentado mulheres com CA $>88 \mathrm{~cm}$ e homens CA $>102 \mathrm{~cm}$ (REZENDE et al., 2006).

${ }^{\circ}$ Considerou como ponto de corte respectivamente RCQ $\leq 0,85$ e RCQ $\leq 1,0$ para mulheres e homens (GOSTON e MENDES, 2001).

${ }^{\mathrm{p}}$ Considerou como ponto de corte o valor $\geq 0,5$ para ambos os gêneros (HSIEH e MUTO, 2005).

${ }^{\mathrm{q}}$ Os pontos de corte considerados para pressão alta consideraram os valores preconizados pelo Joint Committee on Detection, Evaluation and Treatment of High Blood Pressure, que estipula valor $\geq$ a $140 \mathrm{~mm} / \mathrm{Hg}$ para pressão sistólica alta e $\geq$ a $90 \mathrm{~mm} / \mathrm{Hg}$ para pressão diastólica alta (JVC VII, 2003) e/ou em uso de drogas anti-hipertensivas (Nascente et al., 2009).

*IMC: índice de massa corporal 
Para avaliação antropométrica e dos níveis pressóricos foram utilizados os seguintes instrumentos: fita métrica inextensível retrátil Fiber Glass®, balança mecânica antropométrica Filizola ${ }^{\circledR}$, estetoscópios Rappaport Premium ${ }^{\circledR} \quad$ e esfigmomanômetros Aneróide Premium®, calibrados e certificados pelo Instituto Nacional de Metrologia, Normalização e Qualidade Industrial (INMETRO).

A medida do peso corporal foi tomada com o sujeito descalço, trajando roupas leves, posicionando-se com os dois pés sobre a balança, distribuindo o peso corporal igualmente sobre as duas pernas e com o olhar fixo num ponto do horizonte. Antes de cada aferição do peso, o examinador tarava a balança, segundo recomendações do fabricante. Para aferir a altura, o sujeito permanecia sobre a balança, com os pés unidos e a cabeça considerando o plano de Frankfurt.

Para avaliação da CA o sujeito portava roupas leves e a região abdominal fora desnudada. O mesmo fora orientado a permanecer na posição ortostática, com os membros superiores estendidos ao longo do corpo. O perímetro da cintura foi medido na menor curvatura localizada entre a última costela e a crista ilíaca, com o cuidado de não comprimir tecidos, sendo a leitura feita no final de uma expiração. Para avaliar a circunferência de quadril posicionou-se a fita métrica ao redor da região do quadril na área de maior protuberância, sem comprimir tal região.

A avaliação dos níveis pressóricos foi realizada com o indivíduo sentado, após 5 minutos de descanso, com a bexiga vazia, e após pelo menos 30 minutos sem ingestão de alimentos, cafeína e/ou consumo de cigarros. Foram realizadas três medidas, desprezandose a primeira. A pressão arterial foi considerada como a média aritmética da segunda e terceira medidas.

A análise estatística se deu pelo software Statistical Package for the Social Sciences - SPSS v.17.0. Para avaliar associação entre NAF e variáveis independentes, realizou-se análise bivariada através do Teste Qui-quadrado considerando associação ao nível de $p \leq 0,20$. Variáveis independentes que se mostraram associadas foram incluídas nas análises de regressão logística múltipla retendo no modelo final aquelas que se associaram com baixo NAF ao nível de $p \leq 0,05$.

Estudo aprovado pelo Comitê de Ética da Associação Educativa do Brasil (SOEBRAS) com parecer consubstanciado $\mathrm{n}^{\circ}$ 19893.

\section{RESULTADOS E DISCUSSÃO}

Identificaram-se na coleta de dados 275 bombeiros militares lotados no batalhão. Desses, 48 foram considerados inelegíveis: 13 
participavam de cursos em outra cidade e 35 estavam de férias. Dos 227 bombeiros considerados elegíveis, 202 consentiram participar do estudo (taxa de resposta de 88,99\%). As análises do teste $\mathrm{K}$ e CCI evidenciaram consistência inter e intra observadores. Os bombeiros apresentaram média de idade de 32,15 anos $\left( \pm 7,12\right.$; $\mathrm{IC}_{95 \%}$ : $31,16-33,14 ; 21-50)$ e média de tempo de serviço: 9,39 anos $\left( \pm 7,51 ; \mathrm{IC}_{95 \%}: 8,34-10,42\right.$; 1,5-29).

Em relação à prevalência do NAF registrou-se que $7,4 \%(n=15)$ dos bombeiros foi classificado como sedentário; $15,3 \%$ $(n=31)$ como irregularmente ativo $A ; 7,0 \%$ $(n=14)$ como irregularmente ativo $B ; 20,3 \%$ $(n=41)$ como ativo e 50,0\% ( $n=101)$ como muito ativo. Desta forma, ao considerar a categorização proposta para este estudo, $68,3 \% \quad(n=138)$ foi categorizado como "fisicamente ativo" e 31,7\% (n=64) como "fisicamente inativo".
$\mathrm{Na}$ análise bivariada identificou-se associação estatisticamente significante entre baixo NAF e as seguintes variáveis independentes: dimensão realização profissional da Síndrome de Burnout $(p=0,169)$; percepção do nível de QV geral $(p=0,064)$; percepção do nível de QV no domínio físico $(p=0,029)$; estado nutricional $(p=0,074)$; razão cintura estatura $(p=0,095)$; posse de outro emprego $(p=0,048)$ e estrutura organizacional $(p=0,101)$.

Na Tabela 2 são apresentados os resultados do modelo final.

Registrou-se maior chance de baixo NAF entre bombeiros com baixa percepção do nível de QV no domínio físico quando comparados aos bombeiros com alta percepção do nível de QV neste domínio e entre bombeiros que relataram ter posse de outro emprego quando comparados àqueles que afirmaram exercer apenas a atividade laboral militar.

TABELA 2 - Análise de regressão logística múltipla, relação entre nível de atividade física, condições de saúde e ocupacionais entre bombeiros militares, interior de MG, 2012 ( $\mathrm{n}=202)$.

\begin{tabular}{|c|c|c|c|}
\hline \multirow[b]{2}{*}{ VARIÁVEIS } & \multicolumn{3}{|c|}{ NÍVEL DE ATIVIDADE FÍSICA } \\
\hline & OR & $\mathrm{IC}_{95 \%}$ & $p$ \\
\hline \multicolumn{4}{|c|}{ CONDIÇÕES DE SAÚDE } \\
\hline \multicolumn{4}{|c|}{ Percepção do nível de QV domínio físico } \\
\hline Alta & 1,00 & & \\
\hline Baixa & 2,06 & $1,10-3,86$ & 0,023 \\
\hline \multicolumn{4}{|c|}{ OCUPACIONAIS } \\
\hline \multicolumn{4}{|c|}{ Posse de outro emprego } \\
\hline Não & 1,00 & & \\
\hline Sim & 2,14 & $1,02-5,32$ & 0,049 \\
\hline
\end{tabular}

Quase um terço dos bombeiros $(31,7 \%)$ foi classificado como "fisicamente inativo". Prevalências inferiores foram encontradas em inquérito com adultos brasileiros $(29,2 \%$ de insuficientemente ativos) (MALTA et al., 2009) e entre policiais militares do Rio de 
Janeiro, onde se identificou que $24,8 \%$ não praticam AF semanalmente (MINAYO, ASSIS e OLIVEIRA, 2011). Prevalências superiores foram registradas entre policiais militares de Feira de Santana -BA- (37,0\% insuficientemente ativos/inativos) (JESUS e JESUS, 2012) e entre policiais civis do Rio de Janeiro onde $46,4 \%$ relatou não praticar $\mathrm{AF}$ semanalmente (MINAYO, ASSIS e OLIVEIRA, 2011).

Profissionais com menor NAF apresentaram maior chance de baixa percepção no domínio físico de QV. Tal domínio compreende aspectos como energia e fadiga, dor e desconforto, sono e repouso. Resultado este que converge com evidências científicas prévias que adotaram distintos delineamentos, populações, faixas etárias e sexos, tendo registrado associações entre NAF e domínio ou função física de QV (PUCCI et al., 2012), documentando assim, a prática de atividade física como impactante na percepção positiva do domínio físico de QV.

Ressalta-se que em estudo prévio, policiais militares e civis apontaram acometimentos que permeiam os aspectos do domínio físico como: fadiga, cansaço, cervicodorsalgias, cefaleias, enxaquecas, poucas horas de sono e repouso (MINAYO, ASSIS e OLIVEIRA, 2011). Todavia há de se considerar a causalidade reversa uma vez que os aspectos que compreendem o domínio físico da percepção de QV, bem como autoestima, autoimagem, recursos financeiros, ambiente físico e transporte são apontados dentre os fatores mais impactantes no NAF (GUIMARÃES e BAPTISTA, 2011).

Bombeiros com posse de outro emprego tiveram maior chance de serem classificados com menor NAF. Resultado que vai de encontro aos de outros estudos que apontaram a jornada de trabalho e consequente indisponibilidade temporal como barreiras para adesão à prática de atividade física entre sujeitos de diversas categorias profissionais e faixas etárias (JESUS e JESUS, 2012). Pesquisa entre militares de Feira de Santana BA identificou que $36,7 \%$ dos militares apontou a jornada de trabalho como barreira para a prática de atividade física. Possível explicação para tal associação seria a baixa remuneração do policial militar, fazendo com que este tenha outros empregos informais para complementar a renda. Outra possível explicação seria a falta de energia, possível ocorrência de dores e mal estar, mau humor relacionadas a uma jornada de trabalho extensiva. Há de se destacar ainda que responsabilidades familiares como criação dos filhos e tarefas domésticas são consideradas uma extensão da jornada profissional, que requerem uma disponibilidade temporal e consequentemente acabam por dificultar a prática da AF (JESUS e JESUS, 2012).

Destaca-se que a avaliação do NAF e das variáveis investigadas é dinâmico. Portanto, causas e efeitos certamente variam 
ao longo do tempo e, sendo este um estudo seccional, não é possível estabelecer uma relação temporal entre as associações observadas. O uso do IPAQ em sua versão curta pode ter limitado o poder de discussão do presente estudo, uma vez que tal versão impossibilita avaliar a prática de atividade física em diferentes domínios comportamentais (trabalho, lazer, atividades domésticas e transporte) limitando a comparabilidade dos resultados registrados com outros estudos que avaliaram tal prática considerando todos os domínios. Todavia os resultados apresentados são confiáveis, frutos de um estudo censitário e com objetivo pioneiro de determinar os fatores relacionadas ao NAF entre bombeiros militares. Os resultados poderão fomentar novas pesquisas,em diferentes localidades, para melhor compreensão do objeto de pesquisa aqui proposto.

\section{CONCLUSÃO}

Em suma, os registros identificados são preocupantes devido à considerável prevalência de baixo NAF, que se mostrou relacionada à baixa percepção do nível de QV no domínio físico e ao fato do bombeiro ter posse de outro emprego. Como devolutiva sugere-se a revisão e/ou construção de política institucional de atenção à saúde, com o intuito de promover a prática de atividade física, considerável estratégia para melhoria dos indicadores de saúde/QV e das condições ocupacionais.

\section{REFERÊNCIAS}

ABEP. Associação Brasileira de Empresas de Pesquisa. Critério de Classificação

Econômica Brasil. São Paulo, 2012.

\section{AMERICAN COLLEGE OF SPORTS MEDICINE. Appropriate physical activity intervention strategies for weight loss and prevention of weight regain for adults. \\ Medicine and Sciece in Sports and Exercise, v. 41, p. 459-471, 2009.}

BARRETO, A. C.; BARBOSA-BRANCO, A. Influência da atividade física sistematizada no estresse e na fadiga dos trabalhadores do restaurante universitário da Universidade de Brasília. Rev Bras Ativ Fis Saúde, v. 5, n. 2, p. 23-29, 2000.

BENEVIDES-PEREIRA, A. M. T. MBI Maslach Burnout Inventory e suas adaptações para o Brasil. In: Congresso da Sociedade Brasileira de Psicologia, 2001, Rio de Janeiro. Anais. Rio de Janeiro: UERJ, 2001.

BRASIL. Ministério do Planejamento, Orçamento e Gestão. Instituto Brasileiro de Geografia e Estatística - IBGE. Censo Demográfico 2010. Disponível em: http://www.sbafs.org.br/public/Painel/midia/i magem/arquivo/Instru\%C3\%A7\%C3\%B5es $\% 20$ ao\%20autores\%2017032014.pdf

DA MOTA, G. R.; Zanesco, A. Leptina, Grelina e Exercício Físico. Arq Bras Endocrinol Metab, v. 51, p. 25-33, 2007.

DA SILVA, W. C. M.; FARAH, B. Q.; RICARTE, G. B. Atividade Física e Fatores Associados emUsuários do Programa Hiperdia de uma Unidade de Saúde da Família do Recife. R Bras Ci Saúde, v. 16, n. 3, p. 385-392, 2012. 
FLECK, M. P. A. et al. Aplicação da versão em português do instrumento abreviado de avaliação da qualidade de vida "WHOQOLbref". Rev Saúde Pública, v. 34, p. 178-183, 2000.

GOSTON, J. L.; MENDES, L. L. Perfil nutricional de praticantes de corrida de rua de um clube esportivo da cidade de Belo Horizonte, MG, Brasil. Rev Bras Med Esporte, v. 17, p. 13-17, 2011.

GUIMARÃES, A. C. A.; BAPTISTA, F. Atividade física habitual e qualidade de vida de mulheres na meia-idade. Rev Bras Med Esporte. v. 17, n. 5, p . 305-309, 2011.

HSIEH, S. D.; MUTO, T. The superiority of waist-to-height ratio as an anthropometric index to evaluate clustering of coronary risk factors among non-obese men and women. PM. v. 40, p. 216-220, 2005.

JESUS, G. M.; JESUS, E. F. A. Nível de atividade física e barreiras percebidas para a prática de atividades físicas entre policiais militares. Rev Bras Ciênc Esporte. v. 31, n. 2, p. 433-438, 2012.

MACEDO, C. S. G. et al. Benefícios do exercício físico para a qualidade de vida. Rev Bras Ativ Fis Saúde. v. 3, n. 8, p. 19,27, 2003.

MALINA, R.M. Physical activity and fitness: pathways from childhood to adulthood. Am J Hum Bio. v. 13, p. 162-172, 2001.

MALTA, D. C et al. Padrão de atividade física em adultos brasileiros: resultados de um inquérito por entrevistas telefônicas, 2006. Epidemiol Serv Saúde. v. 18, n. 1, p. 7-13, 2009.

MINAYO, M. C. S.; ASSIS, S. G.; OLIVEIRA, R. V. C. Impacto das atividades profissionais na saúde física e mental dos policiais civis e militares do Rio de Janeiro (RJ, Brasil). Ciênc Saúde Coletiva. v. 16, n. 4, p. 2199-2209, 2011.
MONTEIRO, J. K. et al. Bombeiros: um olhar sobre a qualidade de vida no trabalho. Psicol Cienc Prof. v. 27, n. 3, p. 554-565, 2007.

NAHAS, M. V.; BARROS, M. V. G.; FRANCALACCI, V. O. O pentáculo de bem-estar: base conceitual para avaliação do estilo de vida de indivíduos ou grupos. Rev Bras Ativ Fis Saúde. v. 5, n. 2, p. 48-59, 2000.

NASCENTE, F. M. N. et al. Hipertensão arterial e sua associação com índices antropométricos em adultos de uma cidade de pequeno porte do interior do Brasil. Rev Assoc Med Bras. v. 55, n. 6, p. 716-722, 2009.

PAN AMERICAN HEALTH ORGANIZATION. Estratégia e plano de ação regional para um enfoque integrado à prevenção e controle das doenças crônicas. Washington: PAHO; 2007. Disponível em:http://www.bvsde.paho.org/texcom/nutrici on/reg-strat-cncdspt.pdf Acessado em outubro de 2014.

PARDINI, R. et al. Validation of the International Physical Activity Questionaire (IPAQ version 6): pilot study in Brazilian young adults. Rev Bras Cien Mov. v. 9, p. 45-51, 2001.

PITANGA, F. J. G.; LESSA, I. Prevalência e fatores associados ao sedentarismo no lazer em adultos. Cad. Saúde Pública. v. 21, n. 3, p. 870-877, 2005.

PITANGA, F. J. G.; LESSA, I. Sensibilidade e especificidade do índice de conicidade como discriminador do risco coronariano de adultos em Salvador, Brasil. Rev Bras Epidemiol. v. 7, p. 259-269, 2011.

PUCCI, G. C. M. F. et al. Associação entre atividade física e qualidade de vida em adultos. Rev Saúde Pública. v. 46, n. 1, p. 166-179, 2012. 
RECHENCHOSKY. L. et al. Estado nutricional e perfil lipídico de crianças. Rev Educ Fis UEM. v. 20, n. 3, p. 431-440, 2009.

REZENDE, F. A. C. et al. Índice de massa corporal e circunferência abdominal: associação com fatores de risco cardiovascular. Arq Bras Cardiol. v. 87, n. 6, p. 728-734, 2006.

SALLIS, J. F.; OWEN, N. Physical activity and behavioral medicine. Thousands Oaks: Sage Publications, 1999.

SANTOS, A. A.; NASCIMENTO

SOBRINHO, C. L. Revisão sistemática da prevalência da síndrome de burnout em professores do ensino fundamental e médio. RBSP. v. 35, p. 299-319, 2011.

TRIBESSAN, S. et al. Atividade física e aspectos sociodemográficos de mulheres idosas. RBSP. v. 33, n. 3, p. 336-348, 2009.

ZAGO, A. S.; ZANESCO, A. Óxido nítrico, doenças cardiovasculares e exercício físico. Arq Bras Cardiol. v. 87, p. 264-270. 